\title{
Assessment of the hybrid status of some Malesian plants using Amplified Fragment Length Polymorphism
}

\author{
R. Kiew, L.L. Teo and Y.Y. Gan
}

\begin{abstract}
R. Kiew ${ }^{1}$, L.L. Teo and Y.Y. Gan ${ }^{2}$ (1 The Herbarium, Singapore Botanic Gardens, Singapore; ${ }^{2}$ Division of Biology, National Institute of Education, Nanyang Technological University, Singapore). Assessment of the hybrid status of some Malesian plants using Amplified Fragment Length Polymorphism. Telopea 10(1): 225-233. Compared with temperate regions where hybrids are common, hybrids reported from the Malesian region are few. In addition, almost none has been the subject of quantitative or experimental study, either because they are rare or inaccessible or are difficult to grow or have long life cycles. Amplified fragment length polymorphism (AFLP) has proved useful in determining whether taxa suggested as hybrids based on their morphological intermediacy between two putative parents are indeed hybrids. The results of AFLP analysis confirmed the hybrid status of taxa in the following genera, Begonia (Begoniaceae), Mangifera (Anacardiaceae) and Nepenthes (Nepenthaceae), while disproving the hybrid status of duku-langsat, Lansium (Meliaceae).
\end{abstract}

\section{Introduction}

Natural hybrids are relatively common in temperate floras, for example Stace (1991) considered that $21.7 \%$ of the native British flora are hybrids, so it is not surprising that some of these species have been intensively studied, both qualitatively and experimentally, for many years.

In contrast, in the Malesian tropics, natural hybridization is very rare and confined to very few genera, such as Cryptocoryne (Araceae), Globba (Zingiberaceae), Nepenthes (Nepenthaceae), Rhododendron (Ericaceae), and a few other isolated instances. For almost all of these, hybrid status has been suggested by their intermediate morphology between two other species that are presumed to be the putative parents. Very few hybrids, e.g. in Globba (Lim 1973), and Begonia (Teo \& Kiew 1999) have been subject to quantitative study using a hybrid index to assess this morphological intermediacy and even fewer have been subject to cytological study, e.g. Globba (Lim 1972).

Experiments involving growing these hybrids and their putative parents and reconstituting the hybrids are nil. This is not surprising because of several factors: knowledge of how to grow these species is often lacking, e.g. in the case of trying to grow deep shade forest plants or for montane plants to survive in the lowlands or crossing experiments of trees involves an unacceptably long time frame or the ratio of botanists to species in the Malesian region is so small that there is not sufficient manpower for detailed experimental work or there is a lack of expertise, e.g. in fields such as cytology.

To overcome these impediments and, in particular, to be able to assess the genotype rather than the phenotype, Amplified Fragment Length Polymorphisms (AFLP) were used to test whether this would be a suitable method for assessing hybrid status and to identify the putative parents. Molecular markers can be used to discover speciesspecific genetic markers for the presumed parental taxa and the distribution of these 
markers in plants of different phenotypes can be examined to ascertain whether they are hybrids or not. AFLP is a DNA-fingerprinting technique developed by Vos et al. (1995). With AFLP, it is possible to obtain information for many loci randomly distributed throughout the genome in a single assay so it is therefore a powerful means of fingerprinting individuals and establishing genetic relationships of plants based on genetic similarity. According to Morell and Rieseberg (1998), due to extinction of parental alleles following the initial hybridization event, additivity in the molecular markers can often only be detected by analysis of multiple loci, thus making AFLP a suitable technique for studying hybridization. In our study, bands specific to each parent were identified and the inheritant patterns of the hybrid samples were studied. Hybrids commonly have an additive inheritant pattern of parental alleles (Crawford 1990). Beisman et al. (1997) have already used AFLP successfully to distinguish a hybrid of Salix (Salicaceae) and its parents as have Suh et al. (1997) for Aconitum hybrids (Ranunculaceae).

The hybrids we assessed covered a range of life forms: trees (Mangifera and Lansium), climbers (Nepenthes) and perennial herbs (Begonia) and habitats (Begonia is montane, the others lowland).

\section{Kuwini, Mangifera odorata Griff. (Anacardiaceae)}

Ding Hou (1978) suggested that kuwini, Mangifera odorata, was a hybrid between the mango (M. indica L.) and the strong-tasting horse mango ( $M$. foetida Lour.). In Malaysia, the horse mango is a wild species that is sometimes grown in villages, while kuwini is a common village fruit tree but has never been confirmed as growing wild.

Leaf samples were taken from 5 Mangifera odorata trees from Sabah and Sarawak, 5 trees of $M$. foetida from Sabah and 10 trees of of different clones of M. indica from Sabah, Sarawak and Singapore. Using AFLP, seven primer pairs were used resulting in 380 unambiguous bands with $84 \%$ polymorphism (Teo et al. 2002), of which 22 were specific to $M$. indica and 28 specific to $M$. foetida. Kuwini had no unique bands but had 16 of the M. indica species-specific bands and 20 of those of $M$. foetida. The AFLP results therefore confirm that $M$. odorata is a hybrid between these two species. Since $M$. indica is an introduced species, this is clearly a case of an anthropogenic hybrid, where geographical isolation has broken down.

Interestingly, the index of similarity showed that kuwini is closer to M. foetida $(76 \%$ similarity) than it is to M. indica $(66 \%)$. In this case, similarity in genotype is manifested in the phenotype, as morphologically it is difficult (if not impossible) to distinguish sterile plants of kuwini from M. foetida (Corner 1988).

\section{Duku-langsat, Lansium domesticum Correa (Meliaceae)}

Duku-langsat is a popular local fruit. Its fruits are morphologically intermediate between those of langsat and duku, which have variously been treated as a single aggregate species, Lansium domesticum (Mabberley 1985) or as distinct species - duku, Aglaia dokoo and langsat, A. domesticum (Kostermans 1966) - or as races of L. domesticum with duku-langsat as a hybrid between them (Corner 1988). While it is difficult to distinguish the trees when they are not fruiting, the fruits are distinct and in fact sell for different prices in the market. Langsat has smaller, ellipsoid, yellow, thin-skinned fruits, which do not split but ooze a lot of latex when broken. Duku is larger, rotund, brown, very thick-skinned, but splits open and has no latex. It also has thicker and sweeter flesh. Duku-langsat is intermediate in size, is round, brownish and has a skin that splits but which is much thinner than that of duku. This is the preferred fruit and the most expensive among the three.

To ensure correct identification of samples, we used the seeds of five fruits per batch bought at several local markets in Peninsular Malaysia and Singapore. Batches of 
langsat were bought in markets in Johore Baru and Yong Peng (Malaysia) and Singapore, and duku-langsat and langsat from markets in Johore Baru and Singapore.

Five primer pairs were used resulting in 269 unambiguous bands. Ten bands were specific to duku and 20 bands were found in all the langsat and duku-langsat samples, while 78 bands were common to all three. Neither langsat nor duku-langsat had any unique bands.

In addition, the genetic similarity between samples was very high: $91.5-100 \%$ for duku and $85.5-100 \%$ for langsat/duku-langsat. This can be ascribed to duku and langsat being apomictic (Prakash et al. 1977). This high level of similarity indicates that langsat, duku and duku-langsat belong to a single species. The conclusion of Mabberley (1985) that they are cultivars of a variable species, L. domesticum, probably best reflects the taxonomic status of these three fruit types. Langsat is considered the wild form as it is a widespread understorey tree.

\section{Nepenthes hybrids (Nepenthaceae)}

Three pitcher plant hybrids that occur naturally in Singapore were investigated: Nepenthes xhookeriana Lindl., which as early as 1928, on morphological intermediacy, had been suggested by Danser as a hybrid between N. rafflesiana Jack and N. ampullaria Jack; N. xtrichocarpa Miq. that Holttum (1940) considered a hybrid between N. gracilis Korth. and N. ampullaria, and the less common hybrid between N. gracilis and N. rafflesiana reported by Tan et al. (1997).

Six primer pairs were used in the AFLP analysis which yielded 372 unambiguous bands of which 74 bands were common to all three species, N. ampullaria, N. gracilis and $N$. rafflesiana. $N$. gracilis had most species-specific bands (34), followed by N. rafflesiana (22) and N. ampullaria (14). N. xtrichocarpa had 22 bands in common with N. gracilis and 14 in common with N. ampullaria but no unique bands, while the third putative hybrid had 20 bands in common with $N$. gracilis and 14 in common with N. rafflesiana and no unique bands. Nepenthes xhookeriana additively inherited 16 bands from N. rafflesiana and 14 from N. ampullaria with one unique band occurring in half the samples that could either be due to unsampled diversity in the parental plants or to a mutation in the hybrid. The AFLP results therefore confirm the hybrid status of these three taxa and confirmed the parents suggested on morphological grounds.

\section{Begonia hybrid (Begoniaceae)}

A natural begonia hybrid between Begonia decora Stapf and B. venusta King is found in several populations at Cameron Highlands, Peninsular Malaysia, where these two species grow sympatrically in upper montane forest. A quantitative study on plants in the hybrid zone showed that there are hybrid swarms displaying an almost complete continuum between the two putative parents from decora-like hybrids to intermediate hybrids to venusta-like hybrids for both morphological and leaf anatomical characters (Teo \& Kiew 1999). In addition, the hybrids were highly fertile both for pollen viability $(91-100 \%)$ and seed viability $(98 \%)$.

For the AFLP analysis, three populations were studied. Two at higher elevations, one where $B$. decora and $B$. venusta grew sympatrically, the other comprising a hybrid swarm; and the third, at a lower elevation comprised B. decora only. B. venusta was not found growing alone. Six primer pairs produced 502 unambiguous bands of which six were species-specific for $B$. decora, while none were specific to $B$. venusta, which was always found growing together with $B$. decora and/or hybrids. This suggests that through introgression $B$. venusta is no longer genetically distinct from the venusta-like hybrids. However, the phenograms produced were instructive for the two populations with hybrids. 
The phenogram (Fig. 1) for the population where both species grew sympatrically (Berinchang) showed that $B$. decora plants clustered with the decora-like hybrids, while the $B$. venusta plants clustered with the venusta-like hybrids. In the second population (Beremban), while ten years ago (1990) a few plants of B. decora were recorded, none could be located in the vicinity when this study was carried out (2000). In the phenogram for this population (Fig. 2), the intermediate hybrids clustered together with the decora-like hybrids, but the separation between the decora-like and venusta-like hybrids was not complete. In this population, it appears that through introgression neither $B$. decora nor $B$. venusta are any longer distinct. That introgression has occurred over several generations is further supported by the presence of unique bands in a few of the hybrids (Fig. 3).

Apart from B. decora growing over a wider altitudinal range (as low as $1200 \mathrm{~m}$ as opposed to 1700-2000 $\mathrm{m}$ altitude for B. venusta), there are no discernable differences in the physical habitat requirements of these two species. Being a hill resort, there is some disturbance by the opening up of roads and pathways though the montane forest but this does not appear to have affected the begonias, which do not invade the more exposed areas. The breakdown in reproductive isolation between these two species is probably due to habitat changes that affect their common pollinator, a Trigona bee. With pathways opened up the pollinators could move unobstructed over greater distances, which would promote hybridization between contiguous populations.

\section{Discussion}

These case studies show that AFLP is a useful method for identifying hybrids and their parents and confirms the hybrid status of the taxa of Mangifera, Nepenthes and Begonia, while disproving the status of duku-langsat as a hybrid. In addition, it shows that AFLP has potential to tackle the problem of species complexes, such as that of Lansium domesticum.

The number of naturally occurring hybrids in the Malesian region is negligible (probably less than 1\%), compared with their frequency in temperate regions (e.g. $21.7 \%$ for the British flora) or even Hawaii with $9.3 \%$ hybrids reported from the flora (Ellstrand et al. 1996).

Various reasons for this have been suggested. One is the observation that in temperate regions many hybrids occur in disturbed or open areas whereas open areas are relatively few in tropical rain forest. However, both rhododendrons and pitcher plants occupy this niche in montane areas where landslips are relatively frequent. For example, Argent et al. (1988) report that on Mt Kinabalu, some rhododendron hybrids form hybrid zones where they are common and abundant. Indeed, pitcher plants and, to a lesser extent rhododendrons, have invaded disturbed man-made areas, such as roadsides. On the other hand, Ashton (1969) ascribed the great rarity of hybrids among Malesian trees in part due to the inability of primary forest species to become established in the exposed conditions of gaps or open areas so that this niche not available to them. Interestingly, hybridization among native secondary forest species, which effectively invade gaps and open areas, has not been recorded at all.

Over recent geological time, however, Malesia has seen drastic fluctuations in climate and changes in sea level that would create widespread open niches (Morley 1999) but there is no evidence that this has caused hybridization followed by speciation.

Hybridization is often associated with lower fertility of the F1 generation. This may be the case in Nepenthes where hybrids are widespread and occur between almost all species, but where they are often found as single or a few plants and are nowhere 


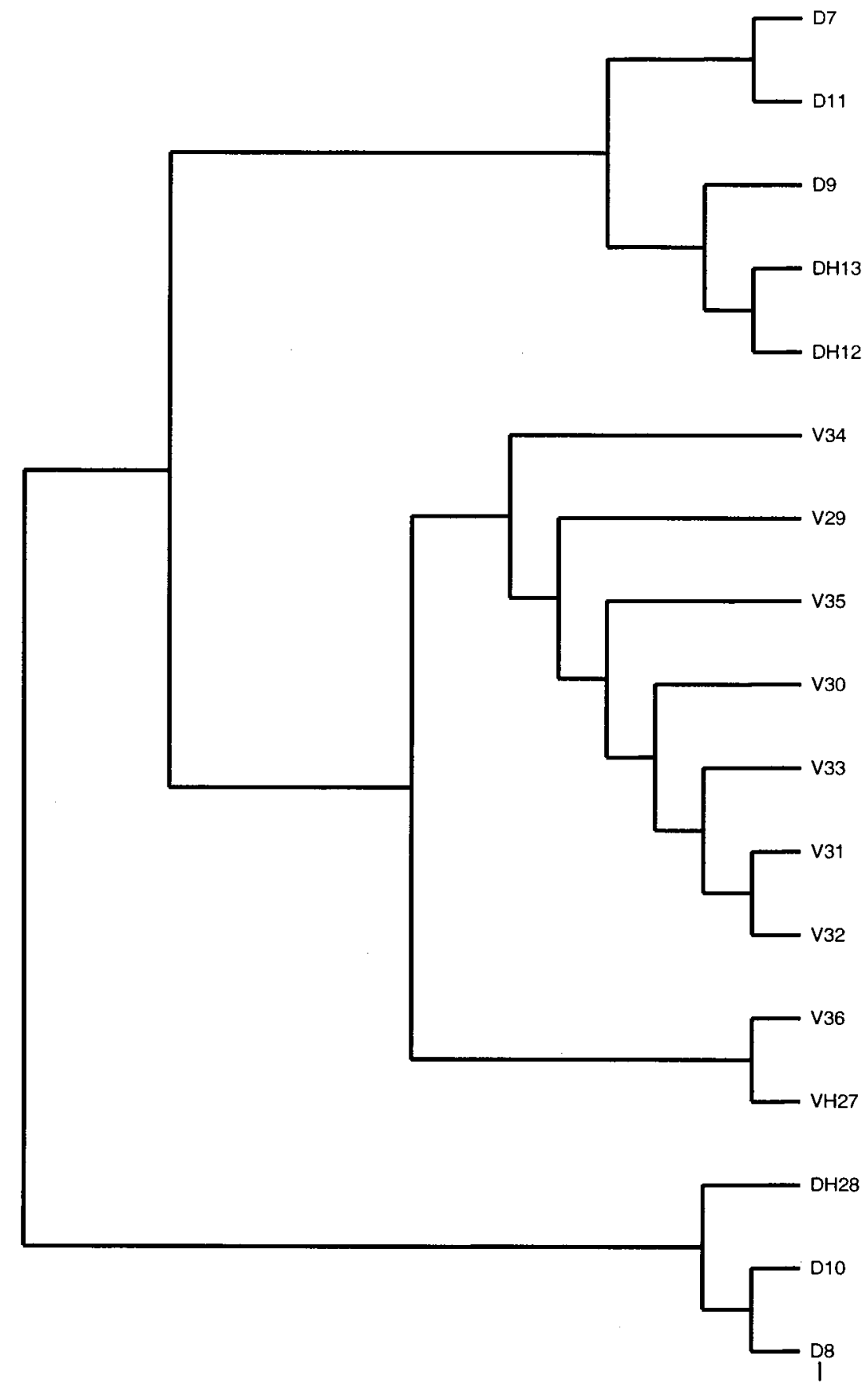

Fig. 1. UPGMA cluster analysis of AFLP data generated by six primer combinations for Begonia samples from Berinchang.

(D:B. decora; DH: decora-like hybrids; V: B. venusta; VH: venusta-like hybrid). 


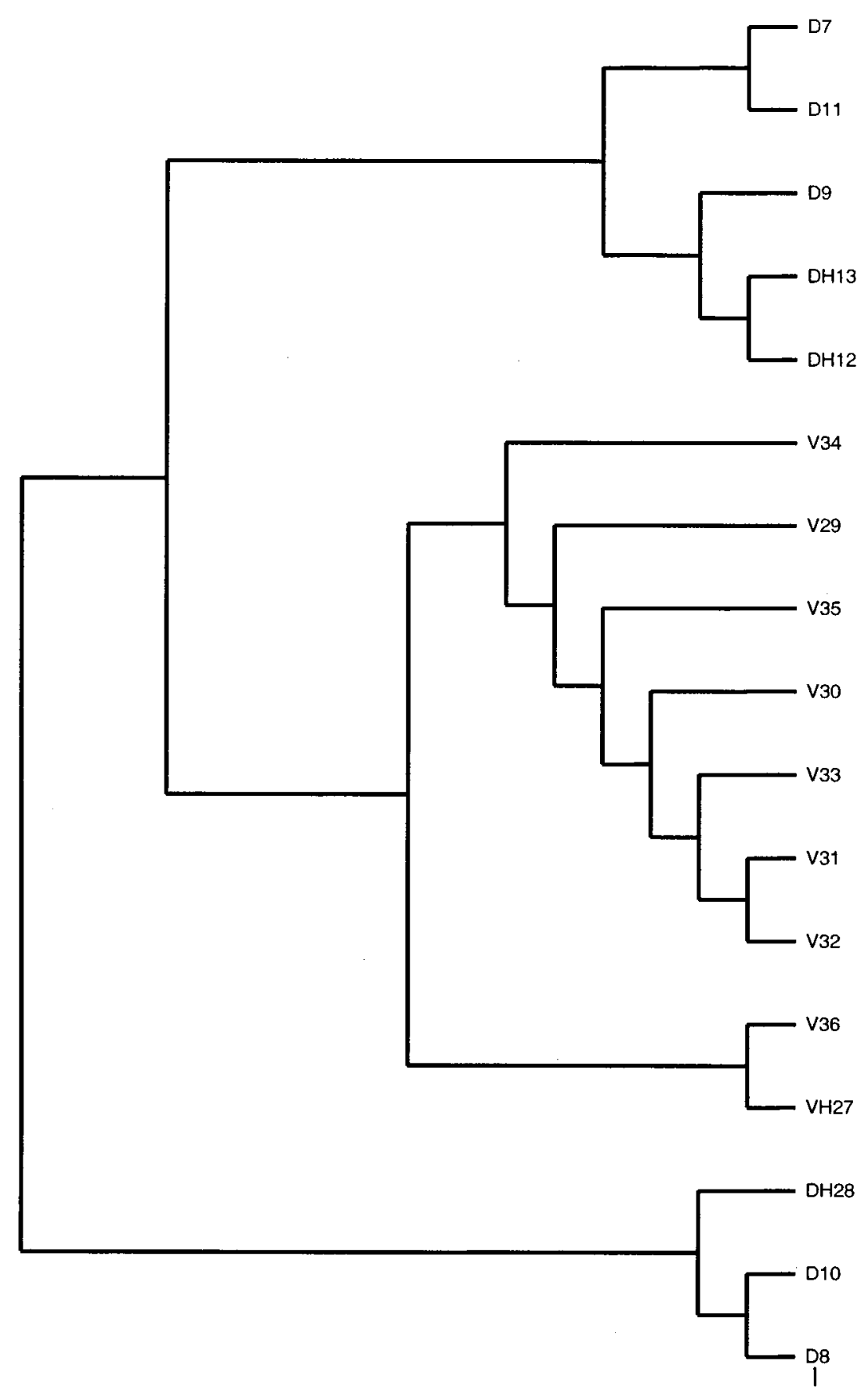

Fig. 2. UPGMA cluster analysis of AFLP data generated by six primer combinations for Begonia samples from Beremban.

(DH: decora-like hybrid; IH: intermediate hybrid; VH: venusta-like hybrid). 


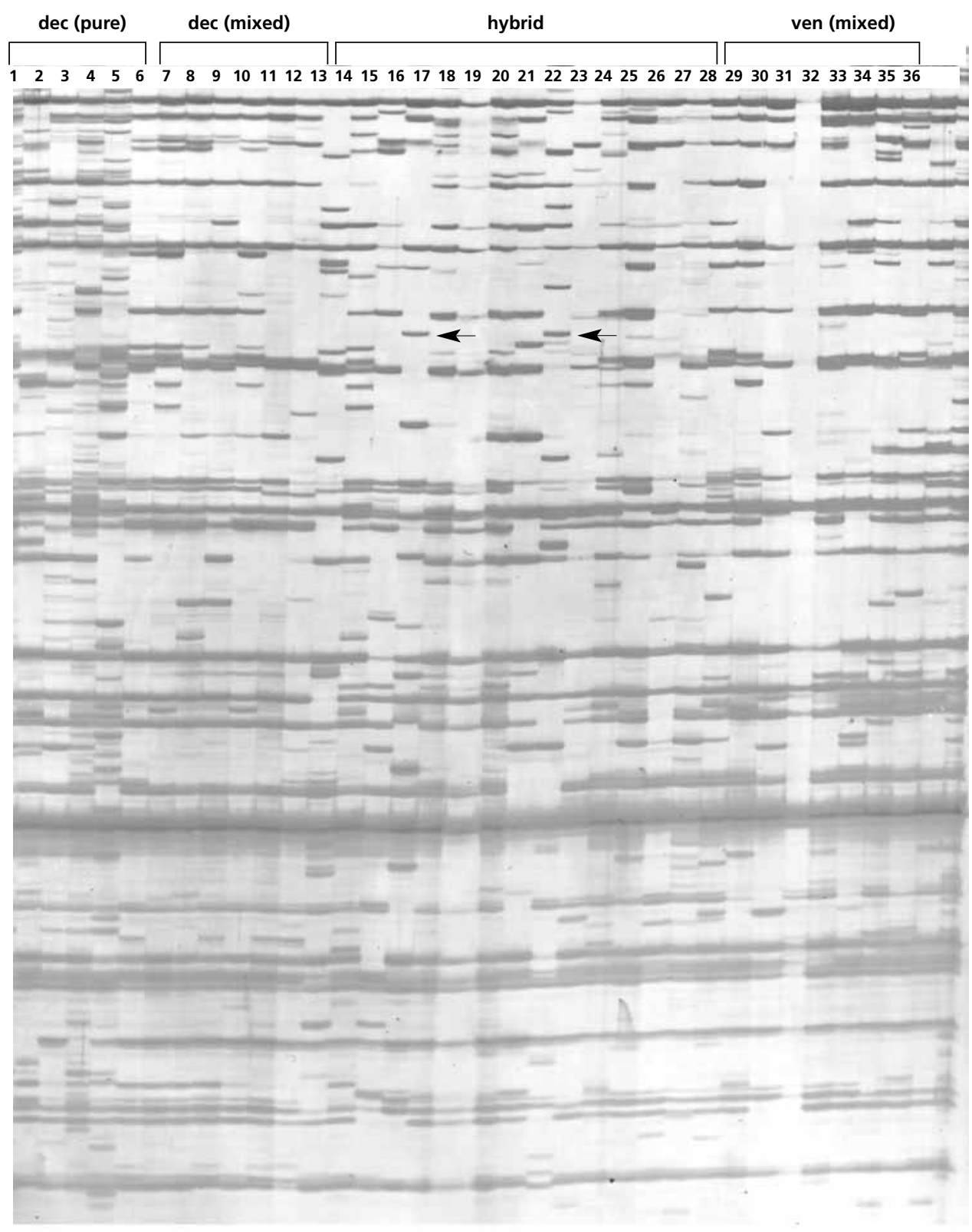

Fig. 3. AFLP profile of begonias using primer pair P10.

(Lanes 1-6: Begonia decora (single species population); lanes 7-13: B. decora (mixed populations); lanes 14-28 hybrids; lanes 29-36 B. venusta (mixed poulations). Arrows indicate unique bands present in the hybrid. 
abundant. Polyploidy is often associated with hybridization as allopolyploidy is one way of overcoming the sterility barrier in the F1 generation. Briggs and Walters (1997) drew attention to the lower occurrence of polyploidy in tropical floras compared with temperate floras and cited the example of $53 \%$ of the British flora being polyploid as compared with only $26 \%$ in West Africa. Levels of polyploidy in the Malesian flora are, however, not known as chromosome numbers for the majority of taxa are still lacking.

A generally held view is that hybrids will be less well adapted to either of the parents' niche so, for example, in conditions of extreme competition in the biodiverse rain forest, the less well adapted hybrids will be selected against. However, Arnold (1997) holds the opposite view as, in his opinion, hybrids are often more fit due, not only to the combination of genotypes from the parents, but also due to the fact that hybridization often produces novel genotypes that may be more fit under certain environmental conditions, particularly disturbed or novel environmental conditions. For species that grow sympatrically, it is often difficult to discern without detailed experimentation what are the critical differences between the niche requirements of the parents.

In the tropics, it may be that pollinator specificity is more important than the physical environment. In this study, the breakdown of reproductive isolation between the two begonia species is more likely to be due to change in pollinator behaviour associated with habitat disturbance than the direct effect of disturbance on the begonias themselves. Clarke (2001) has suggested that in Nepenthes differences in pollinators of the parents may be important in preventing backcrossing between the hybrid and parents.

Another viewpoint suggested by Ashton (1969) for forest trees is that gregarious flowering that is temporally separated between species is also an isolating mechanism that precludes hybridization and is a factor that explains why hybrids are exceedingly rare among rainforest trees.

One thing that is quite clear is that we still know extremely little about the reproductive biology of Malesian plants.

\section{Conclusion}

This study shows that AFLP is a convenient method of analyzing the genotype of putative hybrids and their parents. In addition, the fact that fruit material can be used shows that AFLP has great potential in studying plants where the differences between cultivars and varieties are often defined by fruit characters. However, much still remains unknown about the genotypes and breeding systems of Malesian plants. Our concept of a species is still very much the taxonomic species defined by morphology rather than the biological species. In fact, hybrids have sometimes been invoked when taxa were poorly understood, as in the case of Pentaphragma ellipticum Poulsen, which Airy Shaw (1963) suggested was a hybrid between P. acuminatum Airy Shaw and P. horsfieldii (Miq.) Airy Shaw (Kiew 1989). AFLP provides a convenient method for providing data on hybrid status and can therefore bring us a step closer towards understanding more fully what the taxa we call species really are.

\section{References}

Airy Shaw H.K. (1953) New Malayan species of Pentaphragma. Kew Bulletin 249.

Argent G., Lamb A., Phillipps A., \& Collenette S. (1988) Rhododendrons of Sabah. (Sabah Parks Publications, No 8: Kota Kinabalu, Malaysia). 
Arnold M.L. (1997) Natural Hybridization and Evolution. (Oxford University Press: UK).

Ashton P.S. (1969) Speciation among tropical forest trees: some deductions in the light of recent evidence. Biological Journal of Linnean Society 1: 155-196.

Beismann H., Barker J.H.A., Karp A., \& Speck T. (1997) AFLP analysis sheds light on the distribution of two Salix species and their hybrid along a natural gradient. Molecular Ecology 6: 989-993.

Briggs D., \& Walters S.M. (1997). Plant Variation and Evolution. (Cambridge University Press: Cambridge, UK).

Clarke, C. (2001). Nepenthes of Sumatra and Peninsular Malaysia. (Natural History Publications: Kota Kinabalu, Malaysia).

Corner E.J.H. (1988) Wayside Trees of Malaya. $3^{\text {rd }}$ edn (Malayan Nature Society: Kuala Lumpur, Malaysia).

Crawford D.J. (1990) Plant Molecular Systematics. (J. Wiley: USA).

Danser B.H. (1928) The Nepenthaceae of the Netherlands Indies. Bulletin de Jardin de Botanique Buitenzorg III, 9: 249-438.

Ding Hou (1978) Mangifera. Flora Malesiana 1, 8: 423-440.

Ellstrand N.C., Whitkus R., \& Rieseberg L.H. (1996) Distribution of spontaneous plant hybrids. Proceedings of National Academy of Sciences USA 93: 5090-5093.

Holttum R.E. (1940) Malayan pitcher plants. Malayan Nature Journal 1: 35-44.

Kiew R. (1989) Lowland species of Pentaphragma (Pentaphragmataceae) in Peninsular Malaysia and Singapore. Malayan Nature Journal 43: 1-12.

Kostermans A.J.G.H. (1966) A monograph on Aglaia, sect. Lansium Kosterm. (Meliaceae). Reinwardtia 7: 221-282.

Lim S.N. (1972) Cytogenetics and taxonomy of the genus Globba L. (Zingiberaceae) in Malaya. 1. Taxonomy. Notes Royal Botanic Garden Edinburgh 31: 243-269.

Lim S.N. (1973) Cytogenetics and taxonomy of the genus Globba L. (Zingiberaceae) in Malaya. V. Introgressive hybridization in hexaploids. Journal of Linnean Society, Botany 66: 143-156.

Mabberley D..J. (1985) Meliaceae in Malesia. Blumea 31: 141-143.

Morell P.L. \& Rieseberg L.H. (1998) Molecular test of the proposed diploid hybrid origin of Gilia achilleifolia (Polemoniaceae). American Journal Botany 85: 1439-1453.

Morley R.J. (1999) Origin and Evolution of Tropical Rain Forests. (J. Wiley: USA).

Prakash N., Lim A.L. \& Manurung R. (1977) Embryology of duku and langsat varieties of Lansium domesticum. Phytomorphology 27: 50-59.

Stace C.A. (1991) Plant Taxonomy and Biosystematics. (E. Arnold: UK).

Suh Y., Kim S. \& Park C.W. (1997) AFLP examination for putative hybrids between Aconitum japonicum ssp. napiforme and A. jaluense (Ranunculaceae). Korean Journal of Plant Taxonomy 27: $59-71$.

Tan H.T.M., Tan W.K. \& Wong C.L. (1997). Native species and hybrids - tropical pitcher plants. In: Guide to the Carnivorous Plants of Singapore. (Singapore Science Centre: Singapore).

Teo L.L., Kiew R., Set O., Lee S.K. \& Gan Y.Y. (2002) Hybrid status of kuwini, Mangifera odorata Griff. (Anacardiaceae) verified by amplified fragment length polymorphism. Molecular Ecology 11: 1465-1469.

Teo L.L. \& Kiew R. (1999) First record of a natural begonia hybrid in Malaysia. Gardens' Bulletin Singapore 51: 103-118.

Vos P., Hogers R., Bleeker M., Reijans M., van de Lee T., Hornes M., Frijters A., Pot J., Peleman J., Kupier M. \& Zabeau M. (1995) AFLP: a new technique for DNA fingerprinting. Nucleic Acids Research 23: 4407-4414. 
